\title{
Internal slackening scoring methods
}

\author{
Marco Slikker • Peter Borm • René van den Brink
}

Published online: 13 October 2011

(C) The Author(s) 2011. This article is published with open access at Springerlink.com

\begin{abstract}
We deal with the ranking problem of the nodes in a directed graph. The bilateral relationships specified by a directed graph may reflect the outcomes of a sport competition, the mutual reference structure between websites, or a group preference structure over alternatives. We introduce a class of scoring methods for directed graphs, indexed by a single nonnegative parameter $\alpha$. This parameter reflects the internal slackening of a node within an underlying iterative process. The class of so-called internal slackening scoring methods, denoted by $\lambda^{\alpha}$, consists of the limits of these processes. It is seen that $\lambda^{0}$ extends the invariant scoring method, while $\lambda^{\infty}$ extends the fair bets scoring method. Method $\lambda^{1}$ corresponds with the existing $\lambda$-scoring method of Borm et al. (Ann Oper Res 109(1):61-75, 2002) and can be seen as a compromise between $\lambda^{0}$ and $\lambda^{\infty}$. In particular, an explicit proportionality relation between $\lambda^{\alpha}$ and $\lambda^{1}$ is derived. Moreover, the internal slackening scoring methods are applied to the setting of social choice situations where they give rise to a class of social choice correspondences that refine both the Top cycle correspondence and the Uncovered set correspondence.
\end{abstract}

\footnotetext{
M. Slikker $(\bowtie)$

School of Industrial Engineering, Eindhoven University of Technology, P.O. Box 513, 5600 MB, Eindhoven, The Netherlands

e-mail: M.Slikker@tue.nl

P. Borm

Center and Department of Econometrics and OR, Tilburg University, P.O. Box 90153,

5000 LE, Tilburg, The Netherlands

R. van den Brink

Department of Econometrics, VU University and Tinbergen Institute, De Boelelaan 1105,

$1081 \mathrm{HV}$, Amsterdam, The Netherlands
} 
Keywords Digraphs - Scoring methods - Invariant method · Fair bets method . Social choice correspondences

\section{JEL Classification D71}

\section{Introduction}

Recently, by the application of scoring and ranking methods in search engines on the World Wide Web (such as PageRank in the Google search engine), scoring, and ranking methods for directed graphs regained attention in the literature. For an extensive survey of various scoring methods we refer to Laslier (1997). Kendall (1955) studied a method from Wei (1952), which has come to be called the long path method. Daniels (1969) and Moon and Pullman (1970) reconsidered this method, where Daniels (1969) introduced a normalized version with a better interpretation from a consistency point of view as well. We refer to this method as the normalized long-path method. In addition, Daniels (1969) proposed a different set of fair scores, based on a procedure that has come to be called the Markov method. A recent detailed comparison between the Markov method and the normalized long path method is given by Slutzki and Volij (2006), who refer to them as the fair bets and invariant scoring method, respectively. Finally, Borm et al. (2002) introduced an iterative procedure resulting in the $\lambda$-method.

In this article, we integrate the three scoring methods mentioned above into a single iterative framework. The three methods are shown to be limits of specific iterative procedures. In each of these iterative procedures initially each node in the digraph has an initial score equal to one, and the iteration involves taking the output scores of the previous step as input scores for the next step. We argue that the basic difference between these procedures is by how much in every step of the iteration a node itself shares in the division of its own input score. In fact, we consider a parameterized class of scoring methods $\lambda^{\alpha}$ that are obtained by an iterative procedure where in each step to get (new) output scores, a node shares with a nonnegative weight $\alpha \in(0, \infty)$ in its own current input score, while each of its predecessors shares equally in this with weight one. The boundary cases $\alpha=0$ and $\alpha=\infty$ are defined by considering well-defined limits. We refer to these scoring methods $\lambda^{\alpha}$ with $\alpha \in[0, \infty]$ as internal slackening scoring methods.

The domain of these scoring methods is formed by the class $\mathcal{D}^{N}$ of all irreflexive and connected digraphs (abbreviated to digraphs from now on) on a fixed node set $N$, although we will mainly restrict attention to the subclass $\mathcal{D}_{1}^{N}$ of digraphs with exactly one top cycle. The internal slackening scoring method $\lambda^{1}$ coincides with the $\lambda$-scoring method of Borm et al. (2002). The definitions of the invariant scoring method $I$ and the fair bets scoring method $F$ as proposed in the literature are restricted to subclass $\mathcal{D}_{N}^{N}$ of $\mathcal{D}_{1}^{N}$ of digraphs for which the entire node set $N$ is a top cycle. We show that the internal slackening scoring methods $\lambda^{0}$ and $\lambda^{\infty}$ coincide with $I$ and $F$ on this subclass, respectively, and thus can be viewed as an extension of these methods to a wide range of digraphs. Since $\lambda^{1}$ is the $\lambda$-measure of Borm et al. (2002), this scoring method can be seen as some kind of compromise between the $I$ and $F$ methods. Moreover, on the class $\mathcal{D}_{1}^{N}$, an explicit proportional relation is derived between the various internal 
slackening scoring methods. In particular, the score $\lambda_{i}^{\alpha}$ for a certain node $i$ can be expressed in terms of $\lambda_{i}^{1}, \alpha$, and the number of predecessors of $i$ in the underlying digraph only.

Social choice situations are explicitly considered as an application. Generalizing the result of Borm et al. (2004) with respect to the $\lambda$-scoring method we show that each social choice correspondence associated to an internal slackening scoring method refines both the Top cycle correspondence and the Uncovered set correspondence.

Finally, we highlight some computational aspects of internal slackening scoring methods on arbitrary digraphs in $\mathcal{D}^{N}$, i.e., digraphs with the possibility of multiple top cycles. It is derived that the relative ordering within a specific top cycle does not depend on the digraph structure outside this top cycle. Moreover, the fraction of the score coming from a node not contained in any top cycle to a specific top cycle can be computed explicitly and, interestingly, does not depend on the slackening parameter $\alpha$.

The article is organized as follows. Section 2 recalls basic concepts regarding digraphs and existing scoring methods. Section 3 formally introduces the class of internal slackening scoring methods, discusses relations with the invariant method and the fair bets method, and derives a proportionality result between the internal slackening scoring methods for the class of digraphs with a unique top cycle. Section 4 discusses the application to social choice problems. Section 5 highlights some specific computational aspects for arbitrary digraphs.

\section{Preliminaries: invariant, fair bets, and $\lambda$-scoring methods}

We consider digraphs $(N, D)$ with $N$ a finite set of nodes and $D \subset N \times N$ a binary relation on $N$. When there can be no confusion about the node set we will just write $D$ instead of $(N, D)$. We assume the digraph $D$ to be irreflexive, i.e., $(i, i) \notin D$ for all $i \in N$, and to be connected, i.e., the related undirected graph in which each arc is replaced by an edge is connected. The collection of all such digraphs on $N$ is denoted by $\mathcal{D}^{N}$. In the sequel, we refer to irreflexive and connected digraphs simply as digraphs.

Let $D \in \mathcal{D}^{N}$. $D$ is asymmetric if $(i, j) \in D$ implies that $(j, i) \notin D$ for all $i, j \in N$. $D$ is complete if $\{(i, j),(j, i)\} \cap D \neq \emptyset$ for all $i, j \in N, i \neq j . D$ is a tournament if it is complete and asymmetric. With $i \in N$, the nodes in $S_{D}(i)=\{j \in N \mid(i, j) \in D\}$ are called the successors of $i$ in $D$, and the nodes in $P_{D}(i)=\{j \in N \mid(j, i) \in D\}$ are called the predecessors of $i$ in $D$. The cardinality of these sets is denoted by $s_{D}(i)$ and $p_{D}(i)$, respectively. By $\operatorname{tr}(D) \in \mathcal{D}^{N}$ we denote the transitive closure of $D$, i.e., $(i, j) \in \operatorname{tr}(D)$ if and only if there exists a sequence of nodes $\left(h_{1}, \ldots, h_{t}\right)$ such that $h_{1}=i,\left(h_{k}, h_{k+1}\right) \in D$ for all $1 \leq k \leq t-1$, and $h_{t}=j$. The nodes in $\bar{S}_{D}(i)=S_{t r(D)}(i)$ are called the subordinates of $i$ in $D$. A subset $T \subseteq N$ is a top cycle in $D$ if

(i) $i, j \in T \Rightarrow(i, j) \in \operatorname{tr}(D)$, and

(ii) $i \notin T, j \in T \Rightarrow(i, j) \notin \operatorname{tr}(D)$.

We denote the class of all digraphs with a unique top cycle by $\mathcal{D}_{1}^{N}$. The subclass $\mathcal{D}_{N}^{N}$ contains all digraphs for which $N$ is a top cycle. Note that every tournament belongs to $\mathcal{D}_{1}^{N}$. 
Further, the $N \times N$-adjacency matrix $A^{D}$ with entries $a_{i j}^{D}$ associated with $D$ is defined by:

$$
a_{i j}^{D}= \begin{cases}1 & \text { if }(i, j) \in D \\ 0 & \text { otherwise }\end{cases}
$$

A scoring method with domain $\mathcal{D} \subseteq \mathcal{D}^{N}$ is a function $f: \mathcal{D} \rightarrow \mathbb{R}^{N}$ that assigns a score vector to every digraph in $\overline{\mathcal{D}}$. Scoring method $f: \mathcal{D} \rightarrow \mathbb{R}^{N}$ is efficient if $\sum_{i \in N} f_{i}(D)=|N|$ for all $D \in \mathcal{D}$.

In defining the invariant scoring method $I$ and the fair bets scoring method $F$ on the domain $\mathcal{D}_{N}^{N}$, we follow Slutzki and Volij (2006). The invariant scoring method assigns to a digraph $D \in \mathcal{D}_{N}^{N}$ the unique solution $I(D)$ of the system ${ }^{1}$

$$
\begin{aligned}
& I_{i}(D)=\sum_{j \in N} \frac{a_{i j}}{\sum_{k \in N} a_{k j}} I_{j}(D) \quad \text { for all } i \in N \\
& \sum_{i \in N} I_{i}(D)=|N| .
\end{aligned}
$$

Similarly, the fair bets scoring method assigns to a digraph $D \in \mathcal{D}_{N}^{N}$ the unique solution $F(D)$ of the system ${ }^{2}$

$$
\begin{aligned}
& F_{i}(D)=\sum_{j \in N} \frac{a_{i j}}{\sum_{k \in N} a_{k i}} F_{j}(D) \quad \text { for all } i \in N \\
& \sum_{i \in N} F_{i}(D)=|N|
\end{aligned}
$$

For an interpretation and motivation of these methods we refer to Slutzki and Volij (2006, p. 80).

Finally, we recall the definition of the $\lambda$-scoring method on $\mathcal{D}^{N}$ as introduced by Borm et al. (2002). Let $D \in \mathcal{D}^{N}$. Define $\beta^{0}(D)=e_{N}$, where $e_{N} \in \mathbb{R}^{N}$ is the vector consisting of all ones. Then, for $t \in\{1,2,3, \ldots\}$ define recursively

$$
\beta_{i}^{t}(D)=\sum_{j \in S_{D}(i) \cup\{i\}} \frac{\beta_{j}^{t-1}(D)}{p_{D}(j)+1} \quad \text { for all } i \in N \text {. }
$$

Defining the transition matrix $\Pi^{D}$ as the $N \times N$-matrix with entries $\pi_{i j}^{D}$ given by

$$
\pi_{i j}^{D}=\left\{\begin{array}{cl}
\frac{1}{p_{D}(j)+1} & \text { if }(i, j) \in D \text { or } i=j \\
0 & \text { otherwise, }
\end{array}\right.
$$

\footnotetext{
1 Opposed to most of the literature, we normalize to $|N|$ rather than 1 .

2 Again, we normalize to $|N|$ rather than 1.
} 
Fig. 1 The digraph of Example 2.1

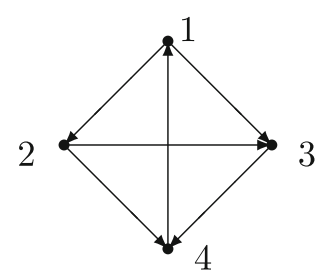

it readily follows that $\beta^{t}(D)=\left(\Pi^{D}\right)^{t} e_{N}$ for all $t \in\{0,1,2, \ldots\}$. The scoring method $\lambda: \mathcal{D}^{N} \rightarrow \mathbb{R}^{N}$ is defined by

$$
\lambda(D)=\lim _{t \rightarrow \infty}\left(\Pi^{D}\right)^{t} e_{N}
$$

It turns out that $\lambda(D)$ is a stationary distribution of $\Pi^{D}$, i.e., $\Pi^{D} \lambda(D)=\lambda(D)$. Moreover, if $D \in \mathcal{D}_{1}^{N}$ then $\Pi^{D}$ has a unique stationary distribution (upon normalization), and thus the stationarity conditions together with the fact that $\sum_{i \in N} \lambda_{i}(D)=|N|$ completely determine $\lambda(D)$.

Example 2.1 Consider the digraph $D \in \mathcal{D}_{N}^{N}$ with $N=\{1,2,3,4\}$ and $D=$ $\{(1,2),(1,3),(2,3),(2,4),(3,4),(4,1)\}$ as represented in Fig. 1.

With

$$
\Pi^{D}=\left[\begin{array}{cccc}
\frac{1}{2} & \frac{1}{2} & \frac{1}{3} & 0 \\
0 & \frac{1}{2} & \frac{1}{3} & \frac{1}{3} \\
0 & 0 & \frac{1}{3} & \frac{1}{3} \\
\frac{1}{2} & 0 & 0 & \frac{1}{3}
\end{array}\right] \text { and } A^{D}=\left[\begin{array}{llll}
0 & 1 & 1 & 0 \\
0 & 0 & 1 & 1 \\
0 & 0 & 0 & 1 \\
1 & 0 & 0 & 0
\end{array}\right]
$$

it follows that $\lambda(D), I(D)$, and $F(D)$ are uniquely determined by efficiency (allocating a total score of 4 ) and the equalities

$$
\begin{gathered}
\left(\Pi^{D}-I_{N}\right) \lambda(D)=0, \\
\left(M^{I}-I_{N}\right) I(D)=0, \\
\left(M^{F}-I_{N}\right) F(D)=0,
\end{gathered}
$$

where

$$
M^{I}=\left[\begin{array}{llll}
0 & 1 & \frac{1}{2} & 0 \\
0 & 0 & \frac{1}{2} & \frac{1}{2} \\
0 & 0 & 0 & \frac{1}{2} \\
1 & 0 & 0 & 0
\end{array}\right], M^{F}=\left[\begin{array}{llll}
0 & 1 & 1 & 0 \\
0 & 0 & 1 & 1 \\
0 & 0 & 0 & \frac{1}{2} \\
\frac{1}{2} & 0 & 0 & 0
\end{array}\right],
$$

and $I_{N}$ is the identity matrix with ones on the main diagonal and zeros off-diagonal. It is found that $\lambda(D)=\frac{4}{23}(8,6,3,6), I(D)=\frac{4}{26}(8,6,4,8)$, and $F(D)=\frac{4}{20}(8,6,2,4)$. 


\section{Internal slackening scoring methods}

In each step of the iterative process underlying the $\lambda$-scoring method, the current score of a node is divided among the node itself and its predecessors. In fact, the node itself receives exactly the same part of its current score as each of its predecessors. This equal treatment of a node and its predecessors was motivated by a game-theoretic perspective in Borm et al. (2002). Here, we generalize this approach and introduce a parameter $\alpha \in[0, \infty]$ representing the sharing weight of a node in his own current score, assuming that all of its predecessors have equal weight, say 1 . Obviously, with respect to the $\lambda$-scoring method, $\alpha=1$.

First, we focus on the case $\alpha \in(0, \infty)$. Let $D \in \mathcal{D}^{N}$. Define $\beta^{\alpha, 0}(D)=e_{N}$ and

$$
\beta_{i}^{\alpha, 1}(D)=\sum_{j \in S_{D}(i)} \frac{\beta_{j}^{\alpha, 0}(D)}{p_{D}(j)+\alpha}+\alpha \frac{\beta_{i}^{\alpha, 0}(D)}{p_{D}(i)+\alpha}
$$

for all $i \in N$. For $\alpha=1$, this measure is studied by van den Brink and Borm (2002). ${ }^{3}$

The following example illustrates the impact of the newly introduced parameter $\alpha$ on the scores of the nodes.

Example 3.1 Consider the digraph $D$ of Example 2.1. Then, for $\alpha \in(0, \infty)$,

$$
\begin{aligned}
\beta^{\alpha, 1}(D)= & \left(\left(\frac{1}{1+\alpha}+\frac{1}{2+\alpha}\right)+\frac{\alpha}{1+\alpha},\left(\frac{1}{2+\alpha}+\frac{1}{2+\alpha}\right)+\frac{\alpha}{1+\alpha},\right. \\
& \left.\left(\frac{1}{2+\alpha}\right)+\frac{\alpha}{2+\alpha},\left(\frac{1}{1+\alpha}\right)+\frac{\alpha}{2+\alpha}\right) \\
= & \left(1+\frac{1}{2+\alpha}, 1+\frac{\alpha}{(1+\alpha)(2+\alpha)}, 1-\frac{1}{2+\alpha}, 1-\frac{\alpha}{(1+\alpha)(2+\alpha)}\right) .
\end{aligned}
$$

Taking the scores provided by $\beta^{\alpha, 1}$ as new input scores on the nodes we obtain $\beta^{\alpha, 2}$ etc. By repeating this procedure, we recursively define for $t \in\{1,2,3, \ldots\}$

$$
\beta_{i}^{\alpha, t}(D)=\sum_{j \in S_{D}(i)} \frac{\beta_{j}^{\alpha, t-1}(D)}{p_{D}(j)+\alpha}+\alpha \frac{\beta_{i}^{\alpha, t-1}(D)}{p_{D}(i)+\alpha}
$$

for all $i \in N$.

Definition 3.1 With $\alpha \in(0, \infty)$ the internal slackening scoring method $\lambda^{\alpha}: \mathcal{D}^{N} \rightarrow$ $\mathbb{R}^{N}$ is determined by

$$
\lambda^{\alpha}(D)=\lim _{t \rightarrow \infty} \beta^{\alpha, t}(D)
$$

for all $D \in \mathcal{D}$.

\footnotetext{
3 We remark that this measure for $\alpha=1$ is different from the original $\beta$-measure considered in van den Brink and Gilles (2000), which, in a restricted setting, comes down to a similar definition with $\alpha=0$.
} 
Along the lines of Borm et al. (2002) it can be shown that $\lambda^{1}$ equals the $\lambda$-scoring method. For the extreme cases $\alpha=0$ and $\alpha=\infty, \lambda^{\alpha}$ will be defined separately later on.

For $D \in \mathcal{D}^{N}$ it follows that $\lambda^{\alpha}$ is a solution to the following system of equations:

$$
\begin{aligned}
& \lambda_{i}^{\alpha}(D)=\sum_{j \in S_{D}(i)} \frac{\lambda_{j}^{\alpha}(D)}{p_{D}(j)+\alpha}+\alpha \frac{\lambda_{i}^{\alpha}(D)}{p_{D}(i)+\alpha} \quad \text { for all } i \in N ; \\
& \sum_{i \in N} \lambda_{i}^{\alpha}(D)=|N|
\end{aligned}
$$

For general $D \in \mathcal{D}^{N}$, the system determined by (1) and (2) does not necessarily have a unique solution.

The iterative process underlying the definition of $\lambda^{\alpha}$ can be seen as a Markov chain with $N \times N$-transition matrix $P^{\alpha}(D)$ (for the exact definition of this matrix we refer to Section 5). As all diagonal elements of $P^{\alpha}(D)$ are positive, all states are aperiodic. For all $D \in \mathcal{D}_{N}^{N}$, i.e., digraphs with $N$ as its unique top cycle, all states are accessible from each other, i.e., the chain is irreducible. It is well-known that a Markov chain on a finite set of states that is aperiodic and irreducible converges to its unique stationary distribution. This in turn implies that for all $D \in \mathcal{D}_{N}^{N}$ the solution of the system determined by (1) and (2) is unique. This result can be extended to $\mathcal{D}_{1}^{N}$ by noting that the relative asymptotic behavior in the top cycle does not change by adding transient states (states not in a top cycle), and all transient states end up with score zero.

In this iterative setting, our newly introduced parameter $\alpha$ has an appealing interpretation. In each step of the procedure above the current scores of the nodes are reallocated. In fact only part of the score of a node remains with this node and the remainder will be distributed (equally) among its predecessors. So, as $\alpha$ increases the internal slackening increases as well.

The following theorem provides a proportionality result between internal slackening scoring methods $\lambda^{\alpha}$ with $\alpha \in(0, \infty)$. It is seen that for $D \in \mathcal{D}_{1}^{N}$ the score $\lambda_{i}^{\alpha}(D)$ can be expressed in terms of $\lambda_{i}^{1}(D)$, the internal slackening parameter $\alpha$, and the number $p_{D}(i)$ of predecessors of $i$. For two vectors $x, y \in \mathbb{R}_{++}^{n}$, we will write $x \sim y$ if there is a $k>0$ such that $x_{i}=k y_{i}$ for all $i \in\{1, \ldots, n\}$.

Theorem 3.1 Let $D \in \mathcal{D}_{1}^{N}$ and let $\alpha \in(0, \infty)$. Then

$$
\lambda^{\alpha}(D) \sim\left(\lambda_{i}^{1}(D) \frac{2\left(p_{D}(i)+\alpha\right)}{(1+\alpha)\left(p_{D}(i)+1\right)}\right)_{i \in N} .
$$

Proof As $D \in \mathcal{D}_{1}^{N}$ and $\alpha \in(0, \infty)$, the system determined by (1) and (2) has a unique solution. Clearly, it suffices to show that the vector $\left(\lambda_{i}^{1}(D) \frac{2\left(p_{D}(i)+\alpha\right)}{(1+\alpha)\left(p_{D}(i)+1\right)}\right)_{i \in N}$ is a solution of (1). Take $i \in N$. Then, 


$$
\begin{aligned}
& \sum_{j \in S_{D}(i)} \frac{\lambda_{j}^{1}(D) \frac{2\left(p_{D}(j)+\alpha\right)}{(1+\alpha)\left(p_{D}(j)+1\right)}}{p_{D}(j)+\alpha}+\alpha \frac{\lambda_{i}^{1}(D) \frac{2\left(p_{D}(i)+\alpha\right)}{(1+\alpha)\left(p_{D}(i)+1\right)}}{p_{D}(i)+\alpha} \\
& \quad=\sum_{j \in S_{D}(i)} \frac{2 \lambda_{j}^{1}(D)}{(1+\alpha)\left(p_{D}(j)+1\right)}+\alpha \frac{2 \lambda_{i}^{1}(D)}{(1+\alpha)\left(p_{D}(i)+1\right)} \\
& =\frac{2}{1+\alpha} \sum_{j \in S_{D}(i)} \frac{\lambda_{j}^{1}(D)}{p_{D}(j)+1}+\frac{2 \alpha}{(1+\alpha)\left(p_{D}(i)+1\right)} \lambda_{i}^{1}(D) \\
& =\frac{2}{1+\alpha}\left(\lambda_{i}^{1}(D)-\frac{2 \alpha}{p_{D}(i)+1}\right)+\frac{\lambda_{i}^{1}(D)}{(1+\alpha)\left(p_{D}(i)+1\right)} \lambda_{i}^{1}(D) \\
& =\frac{2\left(p_{D}(i)+\alpha\right)}{(1+\alpha)\left(p_{D}(i)+1\right)} \lambda_{i}^{1}(D)
\end{aligned}
$$

The third equality follows by (1) for the case $\alpha=1$. We conclude that vector $\left(\lambda_{i}^{1}(D) \frac{2\left(p_{D}(i)+\alpha\right)}{(1+\alpha)\left(p_{D}(i)+1\right)}\right)_{i \in N}$ is a solution of $(1)$.

This completes the proof.

We apply this theorem in the following example.

Example 3.2 Reconsider the digraph $D$ from Example 2.1 and 3.1. Since $\lambda^{1}(D)=$ $\lambda(D)=\frac{4}{23}(8,6,3,6)$ we derive

$$
\lambda^{\alpha}(D) \sim\left(8 \cdot \frac{2+2 \alpha}{2+2 \alpha}, 6 \cdot \frac{2+2 \alpha}{2+2 \alpha}, 3 \cdot \frac{4+2 \alpha}{3+3 \alpha}, 6 \cdot \frac{4+2 \alpha}{3+3 \alpha}\right) .
$$

So, for example, $\lambda^{3}(D) \sim\left(8,6,2 \frac{1}{2}, 5\right)$. As the sum of the scores shared equals 4 , we obtain $\lambda^{3}(D)=\frac{4}{43}(16,12,5,10)$.

Now we turn to the extreme cases $\alpha=0$ and $\alpha=\infty$. Following the lines set out by Definition 3.1 for $\alpha \in(0, \infty)$ will be problematic as will be illustrated in Example 3.4. Note however, that the right-hand side expression in Theorem 3.1 has well-defined limits for $\alpha$ going to 0 or infinity. This leads to the following definition for $\lambda^{0}$ and $\lambda^{\infty}$ on $\mathcal{D}_{1}^{N}$.

Definition 3.2 The internal slackening methods $\lambda^{0}: \mathcal{D}_{1}^{N} \rightarrow \mathbb{R}^{N}$ and $\lambda^{\infty}: \mathcal{D}_{1}^{N} \rightarrow$ $\mathbb{R}^{N}$ are determined by

$$
\begin{aligned}
\lambda_{i}^{0}(D) & :=|N| \cdot \frac{\lambda_{i}^{1}(D) \frac{2 p_{D}(i)}{p_{D}(i)+1}}{\sum_{j \in N} \lambda_{j}^{1}(D) \frac{2 p_{D}(j)}{p_{D}(j)+1}} ; \\
\lambda_{i}^{\infty}(D) & :=|N| \cdot \frac{\lambda_{i}^{1}(D) \frac{2}{p_{D}(i)+1}}{\sum_{j \in N} \lambda_{j}^{1}(D) \frac{2}{p_{D}(j)+1}} .
\end{aligned}
$$

for all $D \in \mathcal{D}_{1}^{N}$ and $i \in N$. 
Fig. 2 The digraph of Example 3.4

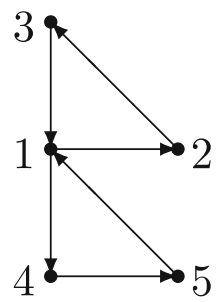

Example 3.3 For the digraph $D$ considered in the previous examples Definition 3.2 leads to

$$
\lambda^{0}(D)=\frac{4}{26}(8,6,4,8) \text { and } \lambda^{\infty}(D)=\frac{4}{20}(8,6,2,4) .
$$

Note that $\lambda^{0}(D)=I(D)$ and $\lambda^{\infty}(D)=F(D)$. In fact, this holds for any $D \in \mathcal{D}_{N}^{N}$ as will be proven in Theorem 3.2.

The definition above for $\alpha$ equal to 0 and for $\alpha$ equal to infinity can be seen as taking limits for $t$ as well as $\alpha$. The following example illustrates that the order of taking limits is relevant.

Example 3.4 Consider the digraph $D \in \mathcal{D}_{N}^{N}$ with $N=\{1,2,3,4,5\}$, and $D=$ $\{(1,2),(1,4),(2,3),(3,1),(4,5),(5,1)\}$ as represented in Fig. 2.

Straightforward calculations show that

$$
\lim _{\alpha \downarrow 0} \beta^{\alpha, t}(D)= \begin{cases}\left(2,1, \frac{1}{2}, 1, \frac{1}{2}\right) & \text { if } t \in\{1,4,7, \ldots\} ; \\ \left(2, \frac{1}{2}, 1, \frac{1}{2}, 1\right) & \text { if } t \in\{2,5,8, \ldots\} ; \\ (1,1,1,1,1) & \text { if } t \in\{3,6,9, \ldots\}\end{cases}
$$

So, $\lim _{t \rightarrow \infty} \lim _{\alpha \downarrow 0} \beta^{\alpha, t}(D)$ does not exist. Using Definition 3.2 one finds that $\lambda_{0}(D)=\left(\frac{5}{3}, \frac{5}{6}, \frac{5}{6}, \frac{5}{6}, \frac{5}{6}\right) .4$

The following lemma provides equations satisfied by the two extreme internal slackening scoring method.

Lemma 3.1 Let $D \in \mathcal{D}_{1}^{N}$.

(i) For all $i \in N$,

$$
\lambda_{i}^{0}(D)=\sum_{j \in S_{D}(i)} \frac{\lambda_{j}^{0}(D)}{p_{D}(j)}
$$

\footnotetext{
${ }^{4}$ Using periodicity arguments one can show that it is not a coincidence that $\lambda^{0}(D)=\frac{1}{3}\left(\left(2,1, \frac{1}{2}, 1, \frac{1}{2}\right)+\right.$ $\left.\left(2, \frac{1}{2}, 1, \frac{1}{2}, 1\right)+(1,1,1,1,1)\right)$.
} 
(ii) If the unique top cycle of $D$ consists of at least two nodes, then for all $i \in N$

$$
\lambda_{i}^{\infty}(D)=\sum_{j \in S_{D}(i)} \frac{\lambda_{j}^{\infty}(D)}{p_{D}(i)}
$$

Proof (i) Let $i \in N$. Put $\delta=\frac{2|N|}{\sum_{j \in N} \lambda_{j}^{1}(D) \cdot \frac{2 p_{D}(j)}{p_{D}(j)+1}}$. From Definition 3.2 and Eq. (1) for $\alpha=1$ we obtain

$$
\begin{aligned}
\sum_{j \in S_{D}(i)} \frac{\lambda_{j}^{0}(D)}{p_{D}(j)} & =\delta \sum_{j \in S_{D}(i)} \frac{\lambda_{j}^{1}(D)}{p_{D}(j)+1} \\
& =\delta\left(\lambda_{i}^{1}(D)-\frac{\lambda_{i}^{1}(D)}{p_{D}(i)+1}\right) \\
& =\lambda_{i}^{0}(D) .
\end{aligned}
$$

(ii) Let $i \in N$. Note that the condition in this part of the lemma implies that $p_{D}(i) \neq 0$. Put $\gamma=\frac{\sum_{j \in N} \lambda_{j}^{1}(D) \cdot \frac{2 p_{D}(j)}{p_{D}(j)+1}}{\sum_{j \in N} \lambda_{j}^{1}(D) \cdot \frac{2}{p_{D}^{(j)+1}}}$. From Definition 3.2 and part (i) we obtain

$$
\begin{aligned}
\sum_{j \in S_{D}(i)} \frac{\lambda_{j}^{\infty}(D)}{p_{D}(i)} & =\frac{\gamma}{p_{D}(i)} \sum_{j \in S_{D}(i)} \frac{\lambda_{j}^{0}(D)}{p_{D}(j)} \\
& =\frac{\gamma}{p_{D}(i)} \lambda_{i}^{0}(D) \\
& =\lambda_{i}^{\infty}(D) .
\end{aligned}
$$

The following theorem illustrates that the two extreme internal slackening scoring methods coincide with the invariant and fair bets scoring methods in case all nodes belong to the top cycle.

Theorem 3.2 Let $D \in \mathcal{D}_{N}^{N}$. Then $\lambda^{0}(D)=I(D)$ and $\lambda^{\infty}(D)=F(D)$.

Proof From Lemma 3.1 (i) it follows for all $i \in N$

$$
\begin{aligned}
\lambda_{i}^{0}(D) & =\sum_{j \in S_{D}(i)} \frac{1}{p_{D}(j)} \lambda_{j}^{0}(D) \\
& =\sum_{j \in N} \frac{a_{i j}^{D}}{\sum_{k \in N} a_{k j}^{D}} \lambda_{j}^{0}(D),
\end{aligned}
$$

where the second equality holds by definition of $A^{D}$. Since $\sum_{i \in N} \lambda_{i}^{0}(D)=|N|$ it follows from the definition of $I(D)$ that $\lambda^{0}(D)=I(D)$. 
It follows from Lemma 3.1 (ii) that, ${ }^{5}$ for all $i \in N$,

$$
\begin{aligned}
\lambda_{i}^{\infty}(D) & =\sum_{j \in S_{D}(i)} \frac{\lambda_{j}^{\infty}(D)}{p_{D}(i)} \\
& =\sum_{j \in N} \frac{a_{i j}^{D}}{\sum_{k \in N} a_{k i}^{D}} \lambda_{j}^{\infty}(D),
\end{aligned}
$$

where the second equality follows from the definition of $A^{D}$. Since $\sum_{i \in N} \lambda_{i}^{\infty}(D)=$ $|N|$ it follows from the definition of $F(D)$ that $\lambda^{\infty}(D)=F(D)$.

\section{An application to social choice situations}

In this section, we apply the internal slackening scoring methods to define a specific class of social choice correspondences. We first recall the basic framework of social choice situations. A social choice situation is a triple $(A, N, p)$ where $A$ is a finite set of agents, $N$ a finite set of alternatives (later on the nodes of a digraph) and the profile $p=\left\{p_{a}\right\}_{a \in A}$ a collection of weak orders on $N$ describing the preference relations of the agents over the set of alternatives. With $i, j \in N$ the notation $i p_{a} j$ means that individual $a$ prefers alternative $i$ to alternative $j$. Throughout we assume that individual preference relations are weak orders, i.e., reflexive, weakly complete, and transitive. Note that a social choice situation $(A, N, p)$ can be identified with the preference profile $p$. The class of all social choice situations $p$ with set of agents $A$ and set of alternatives $N$ is denoted by $\mathcal{S}^{A, N}$.

Although it is straightforward to find the most preferred alternative(s) in a weak order, this is not the case for a preference profile consisting of a collection of such individual preference relations. A social choice correspondence $C$ on a subclass $\mathcal{S} \subseteq \mathcal{S}^{A, N}$ assigns to each $p \in \mathcal{S}$ a non-empty social choice set $C(p)$ of $N$. For surveys on such social choice correspondences we refer to Fishburn (1977) and Laslier (1997).

Given a social choice situation $p \in \mathcal{S}^{A, N}$ the corresponding simple majority win digraph $D_{p} \in \mathcal{D}^{N}$ is defined as follows. With $i, j \in N,(i, j) \in D_{p}$ if and only if $n_{p}(i, j)>n_{p}(j, i)$, where $n_{p}(i, j)=\mid\left\{a \in A \mid i p_{a} j\right.$ and $\left.\neg\left(j p_{a} i\right)\right\} \mid$ is the number of individuals that strictly prefer $i$ to $j$ in the profile $p$.

A social choice correspondence $C$ on $\mathcal{S} \subseteq \mathcal{S}^{A, N}$ is called majoritarian if the social choice set $C(p)$ only depends on the simple majority win digraph $D_{p}$ for all $p \in \mathcal{S}$. Two widely applied majoritarian social choice correspondences are the Top cycle correspondence and the Uncovered set correspondence. Schwartz's Top cycle correspondence TOP (cf. Schwartz (1990)) assigns to every social choice situation $p \in \mathcal{S}^{A, N}$ the union of all top cycles in $D_{p}$. To define the Uncovered set correspon-

\footnotetext{
5 For $|N|=1$, there is nothing to prove. For $|N| \geq 2$ obviously the condition in Lemma 3.1 is satisfied because $D \in \mathcal{D}_{N}^{N}$.
} 
dence we introduce the following terminology. Let $D \in \mathcal{D}^{N}$ and $i, j \in N$. Then we say that node $i$ is covered by $y^{6}$ node $j$ if

(i) $(j, i) \in D$,

(ii) $(i, k) \in D \Rightarrow(j, k) \in D$ for all $k \in N$, and

(iii) $(k, j) \in D \Rightarrow(k, i) \in D$ for all $k \in N$.

The Uncovered set correspondence UNC assigns to every social choice situation $p \in \mathcal{S}^{A, N}$ the set of alternatives that are not covered by any other alternative in $D_{p}$.

Given an internal slackening scoring method $\lambda^{\alpha}$, we define the corresponding majoritarian social choice correspondence $C^{\alpha}$ that assigns to every social choice situation the set of alternatives that have the highest $\lambda^{\alpha}$-score. We restrict attention to the domain $\mathcal{S}_{1}$ of social choice situations for which the corresponding simple majority win digraph has a unique top cycle. ${ }^{7}$ Note that this class contains all social choice situations for which the simple majority win digraph is a tournament: a regular assumption in the social choice literature. Formally, the social choice correspondence $C^{\alpha}$ on $\mathcal{S}_{1}$ is defined by

$$
C^{\alpha}(p)=\left\{i \in N \mid \lambda_{i}^{\alpha}\left(D_{p}\right) \geq \lambda_{j}^{\alpha}\left(D_{p}\right) \quad \text { for all } j \in N\right\}
$$

for all $p \in \mathcal{S}_{1}$.

Borm et al. (2004) showed that the social choice correspondence $C^{1}$ is a refinement of the Top cycle and Uncovered set correspondences. The main result of this section shows that this result can be generalized to any $C^{\alpha}$ with $\alpha \in[0, \infty]$.

Theorem 4.1 Let $p \in \mathcal{S}_{1}$. Then, $C^{\alpha}(p) \subseteq T O P(p)$ and $C^{\alpha}(p) \subseteq U N C(p)$ for all $\alpha \in[0, \infty]$.

Proof For $\alpha=1$ the statements in the theorem follow from Theorem 4.3 in Borm et al. (2004). Moreover, from well-known results on stochastic matrices as discussed in, e.g., Berger (1993) it follows that $\lambda_{i}^{1}\left(D_{p}\right)=0$ for $i \notin T O P(p)$ and $\lambda_{i}^{1}\left(D_{p}\right)>0$ for $i \in T O P(p)$.

Consequently, using Theorem 3.1 for $\alpha \in(0, \infty)$ and Definition 3.2 for $\alpha=0$ and $\alpha=\infty$ it follows for all $\alpha \in[0, \infty]$ that $\lambda_{i}^{\alpha}\left(D_{p}\right)=0$ for all $i \in N \backslash \operatorname{TOP}(p)$, and $\lambda_{i}^{\alpha}\left(D_{p}\right)>0$ for all $i \in \operatorname{TOP}(p)$. Hence, $C^{\alpha}(A, N, p) \subseteq \operatorname{TOP}(A, N, p)$ for all $\alpha \in[0, \infty]$.

Now, let $i \in C^{\alpha}(p)$ and suppose that $i \notin U N C(p)$. Then there is a $j \in N \backslash\{i\}$ with $(j, i) \in D_{p}, S_{D_{p}}(i) \subseteq S_{D_{p}}(j)$ and $P_{D_{p}}(j) \subseteq P_{D_{p}}(i)$. Since $i \in T O P(p)$, also $j \in T O P(p)$, and thus $\lambda_{j}^{\alpha}\left(D_{p}\right)>0$. To shorten notation, abbreviate $D_{p}$ to $D$ and $p_{D_{p}}(k)$ to $p(k)$ for all $k \in N$.

First, consider the case $\alpha \in(0, \infty)$.

\footnotetext{
6 We follow the definition of Borm et al. (2004), which generalizes the definition of Laslier (1997) from tournaments to arbitrary digraphs. Laslier (1997) uses the covering relation that only requires conditions (i) and (ii). Clearly, for tournaments both definitions are equivalent.

7 Section 5 will illustrate that this assumption can be dropped easily.
} 
Then by rearranging terms in (1), for all $r \in N$,

$$
\left(1-\frac{\alpha}{p(r)+\alpha}\right) \lambda_{r}^{\alpha}(D)=\sum_{k \in S_{D}(r)} \frac{\lambda_{k}^{\alpha}(D)}{p(k)+\alpha} .
$$

As

$$
p(j)<p(i) \Rightarrow\left(1-\frac{\alpha}{p(j)+\alpha}\right)<\left(1-\frac{\alpha}{p(i)+\alpha}\right)
$$

and

$$
S_{D}(j) \supseteq S_{D}(i) \cup\{i\} \Rightarrow \sum_{k \in S_{D}(j)} \frac{\lambda_{k}^{\alpha}(D)}{p(k)+\alpha}>\sum_{k \in S_{D}(i)} \frac{\lambda_{k}^{\alpha}(D)}{p(k)+\alpha}
$$

we conclude, $\lambda_{j}^{\alpha}(D)>\lambda_{i}^{\alpha}(D)$, contradicting the fact that $i \in C^{\alpha}(p)$.

Second, consider $\alpha=0$. Using Lemma 3.1 (i) twice we find

$$
\begin{aligned}
\lambda_{j}^{0}(D) & =\sum_{k \in S_{D}(j)} \frac{\lambda_{k}^{0}(D)}{p(k)} \\
& =\sum_{k \in S_{D}(i)} \frac{\lambda_{k}^{0}(D)}{p(k)}+\frac{\lambda_{i}^{0}(D)}{p(i)}+\sum_{k \in S_{D}(j) \backslash\left(S_{D}(i) \cup\{i\}\right)} \frac{\lambda_{k}^{0}(D)}{p(k)} \\
& =\left(1+\frac{1}{p(i)}\right) \lambda_{i}^{0}(D)+\sum_{k \in S_{D}(j) \backslash\left(S_{D}(i) \cup\{i\}\right)} \frac{\lambda_{k}^{0}(D)}{p(k)} \\
& >\lambda_{i}^{0}(D) .
\end{aligned}
$$

Again, this contradicts with the fact that $i \in C^{\alpha}(p)$.

Third, consider $\alpha=\infty$. Since both $i$ and $j$ belong to $\operatorname{TOP}(p)$, Lemma 3.1 (ii) implies

$$
\begin{aligned}
\lambda_{j}^{\infty}(D) & =\sum_{k \in S_{D}(j)} \frac{\lambda_{k}^{\infty}(D)}{p(j)} \\
& =\sum_{k \in S_{D}(i)} \frac{\lambda_{k}^{\infty}(D)}{p(j)}+\frac{\lambda_{i}^{\infty}(D)}{p(j)}+\sum_{k \in S_{D}(j) \backslash\left(S_{D}(i) \cup\{i\}\right)} \frac{\lambda_{k}^{\infty}(D)}{p(j)} \\
& =\left(\frac{p(i)+1}{p(j)}\right) \lambda_{i}^{\infty}(D)+\sum_{k \in S_{D}(j) \backslash\left(S_{D}(i) \cup\{i\}\right)} \frac{\lambda_{k}^{\infty}(D)}{p(j)} \\
& >\lambda_{i}^{\infty}(D) .
\end{aligned}
$$

This contradicts with the fact that $i \in C^{\alpha}(p)$.

So, for all $\alpha \in[0, \infty]$ we may conclude that $i \in C^{\alpha}(p)$ implies that $i \in \operatorname{UNC}(p)$ and hence, $C^{\alpha}(p) \subseteq U N C(p)$ 


\section{Internal slackening scoring methods on $\mathcal{D}^{N}$}

This section highlights some computational aspects of the internal slackening scoring methods $\lambda^{\alpha}, \alpha \in(0, \infty)$, on the general domain $\mathcal{D}^{N}$ of digraphs, i.e., with the possibility of multiple top cycles. Furthermore, the definition of $\lambda^{0}$ and $\lambda^{\infty}$ is extended to $\mathcal{D}^{N}$.

Let $D \in \mathcal{D}^{N}$ and $\alpha \in(0, \infty)$. In line with Borm et al. (2002) for the case $\alpha=1$ the iterative procedure underlying the definition of $\lambda^{\alpha}$ can be explicitly determined by an $N \times N$-transition matrix $P^{\alpha}(D)$ which entries $p_{i j}^{\alpha}(D)$ are given by

$$
p_{i j}^{\alpha}(D)= \begin{cases}\frac{1}{p_{D}(j)+\alpha} & \text { if }(i, j) \in D \\ \frac{\alpha}{p_{D}(j)+\alpha} & \text { if } i=j \\ 0 & \text { otherwise }\end{cases}
$$

Clearly,

$$
\lambda^{\alpha}(D)=\lim _{t \rightarrow \infty}\left(P^{\alpha}(D)\right)^{t} e_{N}
$$

Now let $T$ be a top cycle of $D$. For $j \in N$ define $\rho_{T}^{\alpha}(j)$ to be the probability of arriving in $T$ starting from $j$ according to the stochastic process associated with the transition matrix $P^{\alpha}(D)$ defined above. Furthermore, define $M \subseteq N$ as the set of nodes not contained in any top cycle of $D$. Then $\rho_{T}^{\alpha}(j)$ is uniquely determined by

$$
\rho_{T}^{\alpha}(j)= \begin{cases}1 & \text { if } j \in T \\ 0 & \text { if } j \in N \backslash(M \cup T) \\ \sum_{i \in T} p_{i j}^{\alpha}+\sum_{i \in M} p_{i j}^{\alpha} \rho_{T}^{\alpha}(i) & \text { if } j \in M\end{cases}
$$

The following lemma implies that $\rho_{T}^{\alpha}(j)$ is independent of the explicit choice of the slackening parameter $\alpha$. From this lemma on let $\rho_{T}(j)=\rho_{T}^{\alpha}(j)$ for all $\alpha \in(0, \infty)$.

Lemma 5.1 Let $D \in \mathcal{D}^{N}, T \subseteq N$ a top cycle of $D$, and $\alpha \in(0, \infty)$. Then $\rho_{T}^{\alpha}(j), j \in$ $M$, is uniquely determined by the system

$$
\rho_{T}^{\alpha}(j)=\frac{\left|T \cap P_{D}(j)\right|}{p_{D}(j)}+\frac{1}{p_{D}(j)} \sum_{i \in M \cap P_{D}(j)} \rho_{T}^{\alpha}(i) \quad \text { for all } j \in M \text {. }
$$

Proof By definition $\rho_{T}^{\alpha}(j), j \in M$, is uniquely determined by

$$
\rho_{T}^{\alpha}(j)=\sum_{i \in T} p_{i j}^{\alpha}+\sum_{i \in M} p_{i j}^{\alpha} \rho_{T}^{\alpha}(i) \quad \text { for all } j \in M
$$


equivalently by

$$
\begin{aligned}
\rho_{T}^{\alpha}(j)= & \sum_{i \in T \cap P_{D}(j)} \frac{1}{p_{D}(j)+\alpha}+\sum_{i \in M \cap P_{D}(j)} \frac{1}{p_{D}(j)+\alpha} \rho_{T}^{\alpha}(i) \\
& +\frac{\alpha}{p_{D}(j)+\alpha} \rho_{T}^{\alpha}(j) \quad \text { for all } j \in M,
\end{aligned}
$$

i.e., by

$$
\frac{p_{D}(j)}{p_{D}(j)+\alpha} \rho_{T}^{\alpha}(j)=\frac{\left|T \cap P_{D}(j)\right|}{p_{D}(j)+\alpha}+\frac{1}{p_{D}(j)+\alpha} \sum_{i \in M \cap P_{D}(j)} \rho_{T}^{\alpha}(i) \quad \text { for all } j \in M,
$$

equivalently by

$$
\rho_{T}^{\alpha}(j)=\frac{\left|T \cap P_{D}(j)\right|}{p_{D}(j)}+\frac{1}{p_{D}(j)} \sum_{i \in M \cap P_{D}(j)} \rho_{T}^{\alpha}(i) \quad \text { for all } j \in M .
$$

The next theorem shows that the relative ordering within a specific top cycle does not depend on the digraph structure outside this top cycle. For $D \in \mathcal{D}^{N}$ and a top cycle $T$ of $D$, define the restriction $D_{T} \in \mathcal{D}_{1}^{T}$ by $(i, j) \in D_{T}$ if and only if $(i, j) \in D$ for all $i, j \in T$.

Theorem 5.1 Let $D \in \mathcal{D}^{N}, T$ a top cycle of $D$ and $\alpha \in(0, \infty)$. Then

$$
\lambda_{i}^{\alpha}(D)=\frac{\sum_{j \in N} \rho_{T}(j)}{|T|} \lambda_{i}^{\alpha}\left(D_{T}\right)
$$

for all $i \in T$.

Proof By (1), $\lambda^{\alpha}(D)$ is a solution of the system

$$
x_{i}=\sum_{j \in S_{D}(i)} \frac{x_{j}}{p_{D}(j)+\alpha}+\alpha \frac{x_{i}}{p_{D}(i)+\alpha} \quad \text { for all } i \in N
$$

As $\lambda_{j}^{\alpha}(D)=0$ for all nodes $j$ that are not in a top cycle and nodes in a different top cycle cannot be successor of a node in $T$, we find that $\left(\lambda_{i}^{\alpha}(D)\right)_{i \in T}$ is a solution of the system

$$
x_{i}=\sum_{j \in S_{D}(i) \cap T} \frac{x_{j}}{p_{D}(j)+\alpha}+\alpha \frac{x_{i}}{p_{D}(i)+\alpha} \quad \text { for all } i \in T .
$$


Fig. 3 The digraph of Example 5.1

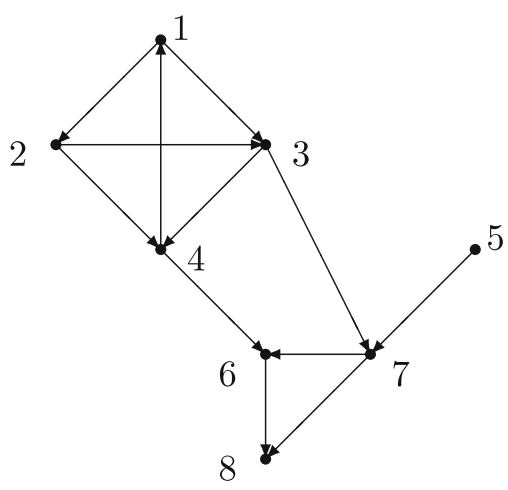

As $S_{D}(i) \cap T=S_{D_{T}}(i)$ and $p_{D}(i)=p_{D_{T}}(i)$ for all $i \in T,\left(\lambda_{i}^{\alpha}(D)\right)_{i \in T}$ is a solution of the system

$$
x_{i}=\sum_{j \in S_{D_{T}}(i)} \frac{x_{j}}{p_{D_{T}}(j)+\alpha}+\alpha \frac{x_{i}}{p_{D_{T}}(i)+\alpha} \quad \text { for all } i \in T
$$

Consequently, $\left(\frac{|T|}{\sum_{j \in N} \rho_{T}(j)} \lambda_{i}^{\alpha}(D)\right)_{i \in T}$ is a solution to (7) as well, while simultaneously $\sum_{i \in T} \frac{|T|}{\sum_{j \in N} \rho_{T}(j)} \lambda_{i}^{\alpha}(D)=\frac{|T|}{\sum_{j \in N} \rho_{T}(j)} \sum_{j \in N} \rho_{T}(j)=|T|$. As (7) corresponds to (1) for $D_{T}$, we conclude that $\left(\frac{|T|}{\sum_{j \in N} \rho_{T}(j)} \lambda_{i}^{\alpha}(D)\right)_{i \in T}$ coincides with the unique solution of (1) and (2) for $D_{T}$. This completes the proof.

On the basis of Lemma 5.1 and Theorem 5.1 we are able to consistently extend the definition of $\lambda^{0}$ and $\lambda^{\infty}$ to the domain $\mathcal{D}^{N}$.

Definition 5.1 Let $D \in \mathcal{D}^{N}$. Then

$$
\lambda_{i}^{0}(D):= \begin{cases}\frac{\sum_{j \in N} \rho_{T}(j)}{|T|} \lambda_{i}^{0}\left(D_{T}\right) & \text { if } i \text { is contained in the top cycle } T \text { of } D \\ 0 & \text { otherwise. }\end{cases}
$$

and

$$
\lambda_{i}^{\infty}(D):= \begin{cases}\frac{\sum_{j \in N} \rho_{T}(j)}{|T|} \lambda_{i}^{\infty}\left(D_{T}\right) & \text { if } i \text { is contained in the top cycle } T \text { of } D \\ 0 & \text { otherwise. }\end{cases}
$$

We conclude by providing two examples.

Example 5.1 Consider the digraph $D \in \mathcal{D}^{N}$ with $N=\{1,2,3,4,5,6,7,8\}$ and $D$ as represented in Fig. 3. 
Fig. 4 The digraph of Example 5.2

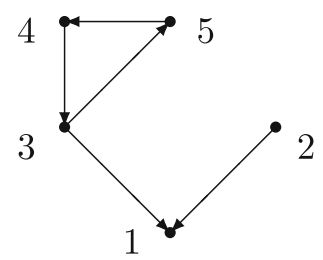

Clearly, there are two top cycles, $T_{1}=\{1,2,3,4\}$ and $T_{2}=\{5\}$. Moreover, solving the system of Lemma 5.1 one finds that $\rho_{T_{1}}(6)=\frac{3}{4}, \rho_{T_{1}}(7)=\frac{1}{2}$, and $\rho_{T_{1}}(8)=\frac{5}{8}$. Consequently, using Theorem 5.1 and Definition 5.1,

$$
\begin{aligned}
\lambda_{T_{1}}^{0}(D) & =\frac{47}{32} \lambda^{0}\left(D_{T_{1}}\right)=\frac{47}{32} \frac{4}{26}(8,6,4,8) \\
\lambda_{T_{1}}^{1}(D) & =\frac{47}{32} \frac{4}{23}(8,6,3,6) ; \\
\lambda_{T_{1}}^{\infty}(D) & =\frac{47}{32} \frac{4}{20}(8,6,2,4) ; \\
\lambda_{5}^{0}(D) & =\lambda_{5}^{1}(D)=\lambda_{5}^{\infty}(D)=\frac{68}{32}
\end{aligned}
$$

while $\lambda_{i}^{0}(D)=\lambda_{i}^{1}(D)=\lambda_{i}^{\infty}(D)=0$ for all $i \in\{6,7,8\}$. Hence, node 5 is ranked first according to $\lambda^{0}$ and $\lambda^{1}$, and second according to $\lambda^{\infty}$.

Example 5.2 Consider the digraph $D \in \mathcal{D}^{N}$ with $N=\{1,2,3,4,5\}$ and $D$ as represented in Fig. 4.

Consider an arbitrary $\alpha \in(0, \infty)$. Node 2 will end up with score $1 \frac{1}{2}$, consisting of his own starting score in the iterative process and half of the starting score of node 1. As node 1 is not in a top cycle he will end up with score 0 . Nodes 3,4 , and 5 equally divide the total score of their top cycle (which includes the other half of the starting score of node 1$)$ resulting in $\lambda^{\alpha}=\left(0,1 \frac{1}{2}, 1 \frac{1}{6}, 1 \frac{1}{6}, 1 \frac{1}{6}\right)$ for all $\alpha \in(0, \infty)$, which is straightforwardly extended to hold for all $\alpha \in[0, \infty]$.

As for $\alpha=2$

$$
\begin{aligned}
\left(\lambda_{i}^{1}(D) \frac{2\left(p_{D}(i)+\alpha\right)}{(1+\alpha)\left(p_{D}(i)+1\right)}\right)_{i \in N} & =\left(0,2,1 \frac{1}{6}, 1 \frac{1}{6}, 1 \frac{1}{6}\right) \nsim\left(0,1 \frac{1}{2}, 1 \frac{1}{6}, 1 \frac{1}{6}, 1 \frac{1}{6}\right) \\
& =\lambda^{\alpha}(D) .
\end{aligned}
$$

we conclude that the proportionality result of Theorem 3.1 cannot be extended to $\mathcal{D}^{N}$. We stress that the proportionality result does hold per top cycle.

\section{Concluding remarks}

We dealt with the ranking problem of the nodes in a directed graph and introduced a class of scoring methods for directed graphs, indexed by a single nonnegative parameter $\alpha$. 
For directed graphs with all nodes in the unique top cycle the scoring methods for $\alpha=0$ and $\alpha=\infty$ coincide with the well-known invariant scoring method and fair bets scoring method (cf. Slutzki and Volij (2006)), respectively. As our scoring methods are defined on the set of all connected digraphs, these two extreme internal slackening scoring methods provide natural unique extensions of both fair bets and invariant scoring methods to directed graphs with several top cycles. For $\alpha=1$ the scoring method corresponds with the existing $\lambda$-scoring method of Borm et al. (2002) and can be seen as a compromise between $\lambda^{0}$ and $\lambda^{\infty}$.

Existing scoring methods generally focus on ranking within a top cycle. Our methods allow comparisons between top cycles as well. In doing so, the total score of the nodes in a top cycle is shown to be independent of the specific internal slackening scoring method selected.

In addition, we provided an explicit proportionality relation between $\lambda^{\alpha}$ and $\lambda^{1}$ for directed graphs with a unique top cycle. This facilitates comparison between the results of the different internal slackening scoring methods and the well-known methods they were shown to coincide with. For general digraphs this proportionality relation does not hold for the full set of nodes, even though it holds per top cycle.

Finally, we introduced social choice correspondences with respect to the scoring methods presented. Each of these correspondences is shown to be a refinement of two correspondences from the literature, namely the Top cycle and Uncovered set correspondences.

Open Access This article is distributed under the terms of the Creative Commons Attribution Noncommercial License which permits any noncommercial use, distribution, and reproduction in any medium, provided the original author(s) and source are credited.

\section{References}

Berger, M. (1993). An Introduction to Probability and Stochastic Processes. New York: Springer.

Borm, P., van den Brink, R., Levinsky, R., \& Slikker, M. (2004). On two new social choice correspondences. Mathematical Social Sciences, 47(1), 51-68.

Borm, P., van den Brink, R., \& Slikker, M. (2002). An iterative procedure for evaluating digraph competitions. Annals of Operations Research, 109(1), 61-75.

Daniels, H. (1969). Round-robin tournament scores. Biometrika, 56(2), 295-299.

Fishburn, P. (1977). Condorcet social choice functions. SIAM Journal on Applied Maths, 33(3), 469-489.

Kendall, M. (1955). Further contributions to the theory of paired comparisons. Biometrics, 11, 80-93.

Laslier, J. (1997). Tournament Solutions and Majority Voting. Berlin: Springer.

Moon, J., \& Pullman, N. (1970). On generalized tournament matrices. SIAM Review, 12, 384-399.

Schwartz, T. (1990). Cyclic tournaments and cooperative majority voting: a solution. Social Choice and Welfare, 7, 19-29.

Slutzki, G., \& Volij, O. (2006). Scoring of web pages and tournaments-axiomatizations. Social Choice and Welfare, 26, 75-92.

van den Brink, R., \& Borm, P. (2002). Digraph competitions and cooperative games. Theory and Decision, 53, 327-342.

van den Brink, R., \& Gilles, R. (2000). Measuring domination in directed networks. Social Networks, 22, 141-157.

Wei, T. (1952). The algebraic foundation of ranking theory. Ph.d. Thesis, Cambridge: Cambridge University. 